\title{
Maturation of Long-Term Potentiation Induction Rules in Rodent Hippocampus: Role of GABAergic Inhibition
}

\author{
Rhiannon M. Meredith, Anna M. Floyer-Lea, and Ole Paulsen \\ University Laboratory of Physiology, Oxford University, Oxford, OX1 3PT, United Kingdom
}

\begin{abstract}
The induction rules of synaptic plasticity are important for the functional operation of a neural network. We asked whether such synaptic plasticity rules change during development from juvenile to adult animals. Using perforated patch and whole-cell recordings from CA1 pyramidal cells in hippocampal slices, we demonstrate here that the postsynaptic requirements for induction of associative long-term potentiation (LTP) shift gradually. Presynaptic stimulation paired with single postsynaptic action potentials became progressively less effective at inducing LTP with advancing developmental age until, in adult hippocampus, postsynaptic bursts of action potentials were necessary to induce synaptic potentiation. This developmental change might be accounted for by changes in $\mathrm{GABA}_{\mathrm{A}}$ receptor-mediated inhibition known to occur in the hippocampus during this postnatal period, because blocking $\mathrm{GABA}_{\mathrm{A}}$ receptor-mediated inhibition re-established the effectiveness of single postsynaptic action potentials at inducing LTP in adult hippocampus. These data reveal a gradual shift in the induction rules for LTP, explained by a maturational change in GABAergic inhibition, and could have implications for our understanding of the role of inhibition in information processing in the brain.
\end{abstract}

Key words: rat; mouse; hippocampus; CA1; development; synaptic plasticity; long-term potentiation; inhibition; $\mathrm{GABA}_{\mathrm{A}}$ receptor; Hebbian; associative LTP

\section{Introduction}

The rules of synaptic plasticity are fundamental to the functional properties of a neural network. Synaptic modifications are important during development, when the network architecture is established and refined. They are also likely to play a physiological role within more mature networks of the brain; for example, synaptic plasticity has been proposed as the main cellular mechanism underlying learning (Ramon y Cajal, 1894; Hebb, 1949; Martin et al., 2000). However, it is not known whether the rules for such synaptic modifications change or remain unaltered during maturation of the network.

Long-term potentiation (LTP) of synaptic transmission remains the most widely studied example of synaptic plasticity (Bliss and Collingridge, 1993). Although conventionally induced by strong high-frequency afferent stimulation, more recently, LTP has been induced by pairing low-frequency presynaptic stimulation with postsynaptic action potentials (Wigström et al., 1986; Magee and Johnston, 1997; Markram et al., 1997), in line with the predictions made by Hebb (1949). Recent studies have emphasized the importance of temporal order for pairinginduced LTP, whereby the postsynaptic spike must occur within a time window of $\sim 20 \mathrm{msec}$ after the presynaptic stimulus for

\footnotetext{
Received June 27, 2003; revised Sept. 29, 2003; accepted 0ct. 3, 2003.

This work was supported by European Commission Framework V Grant QLG3-CT-1999-00192, the Wellcome Trust, and the Biotechnology and Biological Sciences Research Council.

Correspondence should be addressed to Dr. Rhiannon M. Meredith, University Laboratory of Physiology, Oxford University, Parks Road, 0xford, 0X13PT, UK. E-mail: rhiannon.meredith@physiol.ox.ac.uk.

A. M. Floyer-Lea's present address: Oxford Centre for Functional Magnetic Resonance Imaging of the Brain, John Radcliffe Hospital, Headington, 0xford, 0X3 9DU, UK.

Copyright $\odot 2003$ Society for Neuroscience $\quad$ 0270-6474/03/2311142-05\$15.00/0
}

potentiation to occur, whereas reversing the order of presynaptic and postsynaptic activity leads to long-term depression (LTD) (Markram et al., 1997; Bi and Poo, 1998; Debanne et al., 1998). Such spike-timing-dependent plasticity (STDP) (Song et al., 2000) plays an important role in synaptic modification in the developing CNS, first demonstrated in the frog tectum, where pairing of single presynaptic and postsynaptic action potentials is sufficient for synaptic modification to occur (Zhang et al., 1998). However, it is not clear whether similar induction paradigms give rise to synaptic plasticity in the adult brain. Previous studies have reported that postsynaptic burst spiking is necessary to induce LTP using a theta-burst paradigm in adult hippocampal slices from mice and rats (Thomas et al., 1998; Pike et al., 1999). These results raise the question of whether the rules of LTP induction change during postnatal development. To investigate whether the postsynaptic requirement for pairing-induced LTP changes in the hippocampus from juvenile to adult rodents, we compared a pre-before-post-associative LTP induction protocol using either postsynaptic single spikes or bursts of spikes in hippocampal slices at different ages.

Because GABAergic inhibition is an important regulatory factor during induction of LTP (Wigström and Gustafsson, 1983; Davies et al., 1991; Paulsen and Moser, 1998) and GABAergic inhibition gradually increases during the same period of development (Zhang et al., 1991; Cohen et al., 2000; Ganguly et al., 2001; Banks et al., 2002), we also tested whether blocking $\mathrm{GABA}_{\mathrm{A}}$ receptor-mediated inhibition in the adult hippocampus influenced the induction of synaptic plasticity. Together, our data suggest that LTP induction rules change during postnatal development, and that the increase in GABAergic inhibition with mat- 
$\mathrm{Ai}$

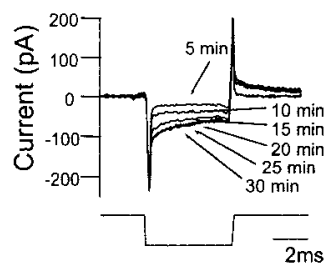

Aii
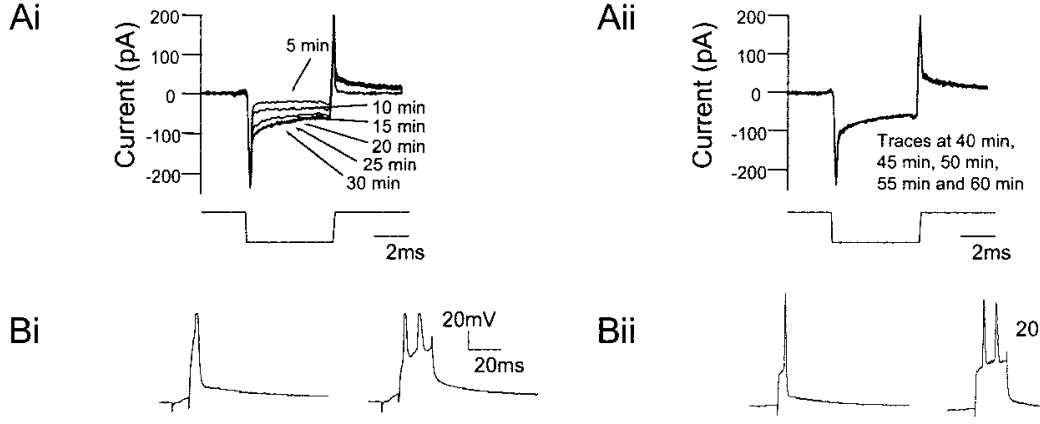

Bii

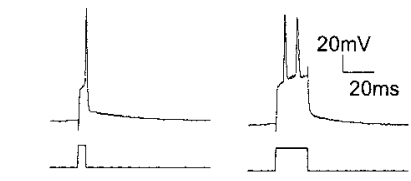

$\mathrm{Ci}$
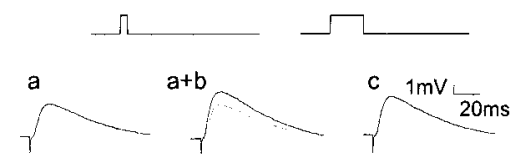

Cii
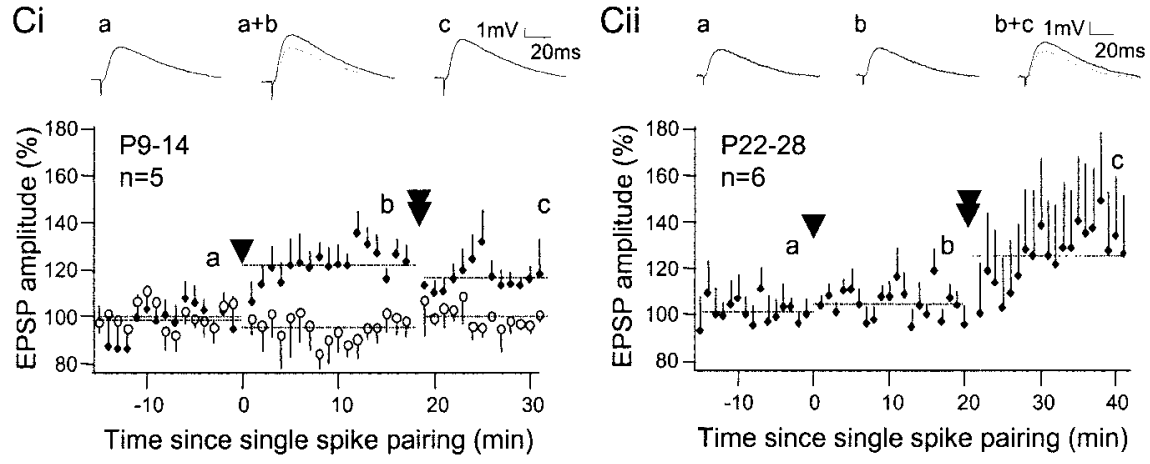

Figure 1. Hebbian LTP induction in two different age groups of mice using perforated patch recordings. Ai, Aii, Current response during voltage clamp to negative $10 \mathrm{mV}$ voltage step during development of perforated patch. For this sample cell, access resistance changed from $\sim 400 \mathrm{M} \Omega$ after 5 min to $\sim 80 \mathrm{M} \Omega$ after $20 \mathrm{~min}(A i)$ and remained stable thereafter for the duration of the experiment (Aii). Bi, Bii, LTP induction protocol for both juvenile (P9 -P14) (Bi) and young adult (P22-P28) (Bii) mice. A single EPSP was paired with a single postsynaptic action potential elicited by current injection ( $5 \mathrm{msec}, 0.1-0.85 \mathrm{nA}$ ) repeated 30 times. After $20 \mathrm{~min}$, a single EPSP was paired with a postsynaptic burst ( $20 \mathrm{msec}, 0.1-0.85 \mathrm{nA}$ ). Stimulation artifacts and capacitance transients have been truncated for clarity. Ci, Cii, Normalized EPSP amplitude monitored over time for both juvenile (Ci) and young adult (Cii) mice. EPSP amplitudes were measured and expressed as a percentage of the mean of the amplitude measured during the last 3 min before single-spike pairing. Test inputs are shown with filled diamonds, and control input is shown with open circles. Error bars indicate SEM. Single arrowheads indicate an episode of single-spike pairing; double arrowheads indicate an episode of burst pairing. Inset, Averaged EPSP waveforms over 3 min periods from baseline period, 20 min after a single-spike pairing episode and 20 min after a burst-pairing episode from a typical cell, are plotted for both age groups of mice. Dotted traces of baseline waveforms are overlaid for direct comparison when potentiation has occurred. Stimulation artifacts have been truncated.

uration of the network alters the nature of the postsynaptic signal necessary to induce synaptic plasticity in the adult hippocampus.

\section{Materials and Methods}

Slice preparation. Horizontal hippocampal slices $(300 \mu \mathrm{m})$ were prepared from C57BL/6 mice and Wistar rats of both sexes at a range of different developmental ages, postnatal day 9 (P9)-P45, after decapitation following cervical dislocation or isoflurane-induced anesthesia in accordance with the United Kingdom Animals (Scientific Procedures) Act of 1986. Slices were stored at room temperature in either a submerged-style holding chamber (for perforated patch recordings) or in an interface holding chamber (for whole-cell patch-clamp recordings) before being transferred individually to the recording chamber, which was perfused with artificial CSF (aCSF) containing the following (in $\mathrm{mm}): 126 \mathrm{NaCl}, 3 \mathrm{KCl}, 1.25 \mathrm{NaH}_{2} \mathrm{PO}_{4}, 2 \mathrm{MgSO}_{4}, 2 \mathrm{CaCl}_{2}, 26 \mathrm{NaHCO}_{3}$, and 10 glucose, bubbled with carbogen gas $\left(95 \% \mathrm{O}_{2}-5 \% \mathrm{CO}_{2}\right)$ to $\mathrm{pH}$ 7.2-7.4. To block $\mathrm{GABA}_{\mathrm{A}}$ receptor-mediated inhibition, in some experiments, either $5 \mu \mathrm{m}$ bicuculline methiodide (Sigma, St. Louis, MO) or $10 \mu \mathrm{M}$ 6-imino-3-(4-methoxyphenyl)-1(6H)-pyridazinebutanoic acid hydrobromide (SR95531, gabazine) (Tocris Cookson, Bristol, UK) was added to the perfusate.

Electrophysiological protocol. Whole-cell and perforated patch-clamp recordings were made from CA1 pyramidal neurons of rats and mice, respectively, with an Axoclamp-2B amplifier (Axon Instruments, Union City, CA) in bridge mode, under visual guidance by infrared differential interference contrast microscopy. Slices were superfused at $\sim 2 \mathrm{ml} / \mathrm{min}$ with aCSF at $24-28^{\circ} \mathrm{C}$. Patch pipettes $(4-6 \mathrm{M} \Omega)$ were pulled from standard wall borosilicate tubing. Pipettes were filled with a solution containing the following (in $\mathrm{mm}$ ): 110 potassium gluconate, 40 HEPES, $4 \mathrm{NaCl}, 4$ ATP-Mg, and $0.3 \mathrm{GTP}, \mathrm{pH}$ 7.2-7.3. During perforated patch recordings, the pipette solution also contained 15-40 $\mu \mathrm{g} / \mathrm{ml}$ gramicidin. These cells were held for at least $30 \mathrm{~min}$ before extracellular stimulation started, to ensure access resistance had stabilized (see Fig. 1 Ai,Aii).

Synaptic efficacy was monitored via activation of two afferent Schaffer collateral inputs (50 $\mu \mathrm{sec}, 10-300 \mu \mathrm{A}$ ) alternately stimulated at $0.2 \mathrm{~Hz}$. EPSP amplitudes were monitored for a baseline period of 12-15 min, after which one of the inputs (the test input) was paired with the minimum intracellular current injection (0.1$0.85 \mathrm{nA}, 5 \mathrm{msec}$ ) necessary to elicit a postsynaptic single action potential coincident with or shortly after the peak of the EPSP. This pairing was repeated 30 times. After an additional 20 $\mathrm{min}$, the same input was then paired with intracellular current injections $(0.1-0.85 \mathrm{nA}, 20$ $\mathrm{msec}$ ) to evoke a postsynaptic burst of two or three action potentials, again repeated 30 times. Control inputs were stimulated alternately throughout the experiments but were not paired with postsynaptic action potentials.

Data analysis. Data acquisition and subsequent offline analysis were performed using Igor Pro (WaveMetrics, Lake Oswego, OR), and statistical analysis was performed using SPSS software (SPSS, Chicago, IL). Synaptic efficacy was monitored using EPSP peak amplitudes, but equivalent results were obtained using slope measurements. Changes in synaptic efficacy were estimated by using the EPSP amplitudes expressed as a percentage of the mean EPSP amplitude from either the total baseline or the 3 min baseline period immediately before each pairing episode. All values are given as means \pm SEM unless otherwise stated. Statistical significance was assessed using the Student's two-tailed $t$ test and linear regression analysis.

\section{Results}

\section{Effectiveness of single-spike pairing changes with postnatal age}

To compare the effectiveness of a single-spike pairing protocol at inducing LTP in hippocampal slices from juvenile and young adult mice, perforated patch-clamp recordings were made from visualized CA1 pyramidal neurons (Fig. 1 Ai,Aii). EPSP peak amplitudes were monitored in two pathways stimulated alternately every $5 \mathrm{sec}$. After a stable baseline period of at least $12-15 \mathrm{~min}$, a pairing protocol consisting of a single afferent stimulus in one of the pathways (the test input) followed within $\sim 10 \mathrm{msec}$ by a single postsynaptic action potential was repeated 30 times (Fig. $1 \mathrm{Bi}, \mathrm{Bii})$. The stimulation frequency was unaltered during the pairing. In hippocampal slices from juvenile mice (P9-P14), this single-spike pairing protocol was sufficient to induce inputspecific LTP in the test pathway [EPSP amplitude measured 15 min after pairing: test input, $122 \pm 6 \%$ (mean \pm SEM), $p<0.05$, $n=5$; control input, $100 \pm 7 \%, n=5$ ] (Fig. 1Ci). In contrast, in young adult mice (P22-P28), an identical pairing protocol failed to induce any significant change in synaptic efficacy in the test 
pathway (102 \pm 4\%, 20 min after pairing; $n=6$ ) (Fig. 1Cii). This failure to induce LTP by single-spike pairing was not attributable to a deficient expression mechanism, since an increase in synaptic efficacy was observed after subsequent pairing of presynaptic stimulation with postsynaptic bursting activity $(123 \pm 2 \%, 20$ min after pairing; $p<0.05 ; n=5$ ) (Fig. 1Cii). In addition, the single-spike pairing protocol appeared to saturate LTP in juvenile mice, because in these animals, no additional increase in EPSP amplitude was observed after a subsequent pairing episode of presynaptic stimulation with postsynaptic bursting activity $(99 \pm 1 \%$; $n=3$ ) (Fig. $1 C i$ ).

To investigate whether this apparent age-dependent difference in induction of LTP is robust against changes in recording mode or species, identical pairing protocols were performed with conventional whole-cell patch-clamp recordings in slices from juvenile (P12-P15) and young adult rats $(\mathrm{P} 25-\mathrm{P} 43)$. In the $\mathrm{P} 12-\mathrm{P} 15$ group, 30 repetitive pairings of presynaptic activity followed by single postsynaptic action potentials were sufficient to induce synaptic potentiation $(186 \pm 22 \%, 20 \mathrm{~min}$ after pairing; $p<0.05 ; n=11$ ) (Fig. $2 A)$. A subsequent burst-pairing episode did not cause any additional potentiation (100 \pm $5 \%, 20$ min after pairing; $n=9)$. In contrast, recordings made from $\mathrm{P} 25-\mathrm{P} 43$ rats showed on average a much smaller and nonsignificant increase in synaptic efficacy after the single-spike pairing protocol $(123 \pm 11 \%$, 20 min after pairing; $p>$ $0.05 ; n=11$ ) (Fig. 2 B). However, a robust and statistically significant synaptic potentiation was observed after an episode of 30 pairs of presynaptic stimulation and postsynaptic bursts $(167 \pm 19 \%, 20 \mathrm{~min}$ after pairing; $p<0.05 ; n=10$ ) (Fig. $2 B$ ). The total amount of potentiation at the end of the experiment relative to baseline after both single-spike and burst pairing was similar in the two age groups (193 \pm $30 \%$ in $\mathrm{P} 12-\mathrm{P} 15$ rats vs $185 \pm 8 \%$ in $\mathrm{P} 25-$

$\mathrm{P} 43$ rats), and there was a negative correlation between potentiation after single-spike pairing and additional potentiation after subsequent burst pairing for both age groups combined $(r=$ $-0.51 ; p<0.05 ; n=19)$. Thus, the period of maturation of synaptic plasticity might last longer in rats than in mice, but we conclude that a developmental shift in induction requirement for associative LTP holds for both rats and mice and under conventional whole-cell as well as perforated patch-clamp recording conditions.

\section{Age-dependent profile of LTP induction}

To analyze whether the change in induction rules is a gradual process or occurs abruptly during development, the amount of synaptic potentiation induced by these pairing protocols was compared over a range of different developmental ages in rats (P12-P43) (Fig. 2C,D). The amount of potentiation induced by

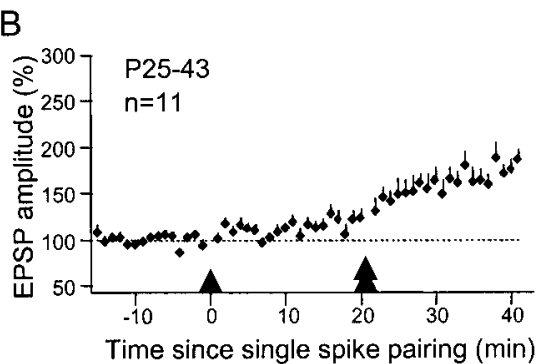

\section{$\mathrm{D}$}

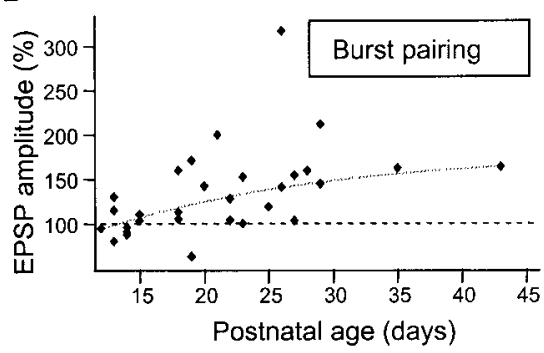

F

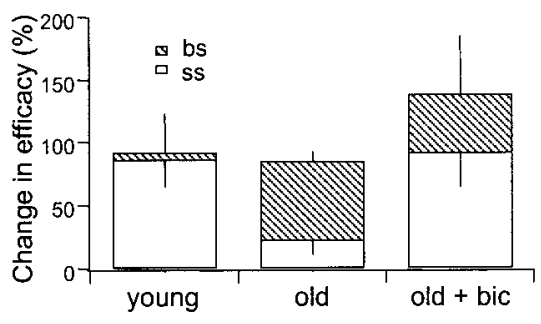

Figure 2. Developmental change in LTP induction in juvenile and young adult rats. $A, B$, Effectiveness of single-spike and burst erdings. Single arrowheads indicate an episode of single-spike pairing; double arrowheads indicate an episode of burst pairing. Er bars indicate SEM. C, D, Normalized peak EPSP amplitude after pairing episode plotted against the developmental age of the (P18-P23). Peak EPSP amplitude was expressed as a percentage of a $3 \mathrm{~min}$ baseline period immediatel and are shown as dotted lines in the graphs. E Effectiveness of single-spike and burst-pairing episodes on EPSP potentiation P30-P45 rats in the presence of $5 \mu \mathrm{m}$ bicuculline methiodide. EPSP amplitudes ( 6 responses averaged per minute) expressed as a percentage of prepairing baseline period were monitored over time using whole-cell patch-clamp recordings. Single arrowheads indicate an episode of single-spike pairing; double arrowheads indicate an episode of burst pairing. Error bars indicate SEM initial baseline responses for hippocampal rat slices taken from groups of younger rats (P12-P15), older rats (P25-P43), and older rats (P30 -P45) in the presence of $5 \mu \mathrm{m}$ bicuculline methiodide (bic). ss, Change in efficacy after single-spike pairing; bs, change in efficacy after burst-pairing episode. Error bars indicate SEM.

the single-spike pairing protocol varied considerably between experiments and within the same age group. However, together, the effectiveness of postsynaptic single-spike activity correlated negatively with developmental age $(r=-0.48 ; p<0.05 ; n=34)$ (Fig. 2C). Conversely, the ability of the burst-spike pairing protocol to induce additional potentiation correlated positively with developmental age $(r=0.48 ; p<0.05 ; n=30)$ (Fig. $2 D)$. Control inputs did not show any significant correlations between developmental age and the amount of synaptic change for either single-spike pairing $(r=-0.3 ; p>0.1 ; n=26)$ or burst-pairing protocols $(r=0.16 ; p>0.1 ; n=22)$. Therefore, during maturation of the hippocampal network, there appears to be a gradual shift in the effectiveness of postsynaptic single spikes to induce input-specific synaptic potentiation such that by young adulthood, postsynaptic bursts become necessary. 
We asked whether changes in membrane potential or width of the action potential during pairing episodes could explain the changes in synaptic plasticity that we observed. However, no significant correlations were seen between membrane potential or spike width (measured at 20 and 50\% of peak spike height) and the amount of synaptic potentiation induced by the single-spike pairing protocol ( $\mathrm{P} 12-\mathrm{P} 43 ; n=34)$. Although these data failed to show any correlation between the somatically recorded spike parameters during pairing and the observed shift in induction rules, developmental changes in the extent of dendritic backpropagation of action potentials cannot be excluded.

\section{Effect of GABAergic inhibition on LTP induction rules in adult hippocampus}

To assess whether the developmental change in induction requirements for LTP could be accounted for by an increased level of GABAergic inhibition in the adult hippocampus, single-spike and burst-pairing protocols were repeated using whole-cell patch-clamp recordings in hippocampal slices taken from young adult rats (P30-P45) in the presence of $5 \mu \mathrm{M}$ bicuculline methiodide. In contrast to our findings during control conditions (Fig. $2 B$ ), the single-spike pairing paradigm induced a significant synaptic potentiation in the presence of the $\mathrm{GABA}_{\mathrm{A}}$ receptor antagonist (192 $\pm 28 \%, 20$ min after pairing; $p<0.05 ; n=5$ ) (Fig. $2 E$ ). An additional burst-pairing episode produced no significant additional potentiation $(123 \pm 11 \%, 20$ min after pairing; $p>0.1$; $n=4)$. Similarly, LTP was also observed after a single-spike pairing protocol in the presence of another specific $\mathrm{GABA}_{\mathrm{A}}$ receptor antagonist, SR95531 (10 $\mu \mathrm{M})$, in hippocampal slices taken from animals of a similar age range $(262 \pm 29 \%, 20 \mathrm{~min}$ after pairing; $p<0.05 ; n=5$; data not shown).

A comparison of the change in synaptic efficacy after singlespike and burst-pairing episodes in hippocampal slices from young rats (P12-P15), old rats (P25-P43), and old rats in the presence of $5 \mu \mathrm{M}$ bicuculline methiodide (P30-P45) confirmed a significant difference in the level of potentiation after single-spike pairing between old rats in the absence and presence of the $\mathrm{GABA}_{\mathrm{A}}$ receptor antagonist (without bicuculline, $23 \pm 11 \%, n=$ 11 ; with bicuculline, $92 \pm 28 \%, n=5$; $p<0.05$ ). Moreover, although the single-spike pairing protocol did not induce a significant potentiation in old rats (P25-P43) under control conditions, there was no significant difference in the total change in synaptic efficacy after both single-spike and burst pairing for old rats compared with young rats (P12-P15, $93 \pm 30 \%, n=9$; $\mathrm{P} 25-\mathrm{P} 43,85 \pm 8 \%, n=10 ; p>0.5)$.

\section{Discussion}

We demonstrated here that there is a developmental shift in the effectiveness of a single-spike pairing protocol at inducing LTP in the CA1 subfield of hippocampus. The amount of synaptic potentiation induced by single-spike pairing correlates negatively with developmental age. Thus, in hippocampal slices from young rodents (P9-P15), repeated pairings of presynaptic stimuli with single postsynaptic action potentials are sufficient for LTP induction, whereas in older rodents (P22-P43), postsynaptic burst activity appears to be necessary to induce similar increases in synaptic efficacy. Furthermore, in rat hippocampi from a range of developmental ages (P12-P43), the total synaptic potentiation after both single-spike and burst pairing was similar in the younger and older groups, and single-spike pairing was negatively correlated with any additional potentiation after burstspike pairing. We also investigated whether an alteration in GABAergic inhibition could underlie this developmental change in induction rules, and report that under conditions of blocked $\mathrm{GABA}_{\mathrm{A}}$ receptor-mediated inhibition, a single-spike pairing protocol alone can induce LTP in the adult hippocampus. This potentiation is comparable in magnitude to that observed in the young hippocampus, as well as to the total potentiation after burst pairing in the adult hippocampus under control conditions.

STDP, whereby single presynaptic and postsynaptic action potentials are paired in a relative order within a narrow time window, has been observed in a variety of different preparations (Paulsen and Sejnowski, 2000; Bi and Poo, 2001). In the hippocampus, previous STDP experiments used dissociated culture prepared from embryonic day 18-20 rats or "organotypic" culture prepared from early postnatal rats (P5-P7) (Bi and Poo, 1998; Debanne et al., 1998). Our findings in rodents up to 2 weeks of age are in agreement with these studies. However, our results show that as the hippocampus matures, single postsynaptic action potentials within this pairing protocol become progressively less effective at inducing LTP. One study has reported the induction of LTP using presynaptic stimuli paired with single postsynaptic action potentials at $5 \mathrm{~Hz}$ in CA1 hippocampus of P26-P33 rats (Nishiyama et al., 2000). However, the authors used cesium methanesulfonate and tetraethylammonium chloride in the recording pipette, thus blocking $\mathrm{K}^{+}$channels, which would increase the width of the postsynaptic action potential and, in effect, depolarize the postsynaptic membrane more effectively than perhaps would be observed under more physiological conditions.

Postsynaptic burst activity has been observed previously to be necessary for induction of LTP using a theta-frequency stimulation paradigm with both extracellular and microelectrode recordings in adult mouse and rat hippocampus in vitro, respectively (Thomas et al., 1998; Pike et al., 1999). Our findings confirm and extend those data with whole-cell and perforated patch recordings by showing that for an STDP paradigm in the young adult hippocampus, postsynaptic burst activity is sufficient for synaptic plasticity when paired with single afferent stimuli; we also show that no change from the baseline frequency of presynaptic stimulation is required.

What is the cellular mechanism underlying the shift in induction requirement with developmental age? A developmental transition from a depolarizing to a hyperpolarizing effect of synaptic $\mathrm{GABA}_{\mathrm{A}}$ receptor activation has been reported in hippocampal circuitry during the first postnatal week (Cherubini et al., 1991; Zhang et al., 1991; Ganguly et al., 2001). In the ensuing postnatal weeks, fast $\mathrm{GABA}_{\mathrm{A}}$ receptor-mediated miniature IPSCs increase in frequency and decrease in both amplitude and decay time, with adult-level maturation of such kinetic IPSC properties reached by the third postnatal week, although more subtle changes in $\mathrm{GABA}_{\mathrm{A}}$ receptor-mediated responses to benzodiazepines still develop between adolescent and adult hippocampus (Cohen et al., 2000; Banks et al., 2002). Thus, the transition from a postsynaptic single-spike to burst-pairing protocol may be explained by a gradual maturation in $\mathrm{GABA}_{\mathrm{A}}$ receptor-mediated inhibition in the hippocampus, whereby greater postsynaptic depolarization is necessary for LTP induction in mature synapses.

Although we did not detect any correlation with spike parameters as measured at the soma, there may still be differences in dendritic back-propagation of action potentials between juvenile and adult animals. Because back-propagation of action potentials is under GABAergic inhibitory control (Tsubokawa and Ross, 1996), and back-propagating action potentials again can control dendritic GABAergic input (Morishita and Alger, 2001), there appears to be an intimate relationship between back-propagating 
action potentials, GABAergic inhibition, and synaptic plasticity. Maturational changes in any of these three factors might thus influence the computational properties of the network. In addition to the GABAergic influence on the extent or level of back-propagation of action potentials, the developmental change we observe in STDP rules in CA1 pyramidal cells could also involve changes in $\mathrm{K}^{+}$channel expression. For example, it has been reported in adult rats (5-8 weeks of age) that to boost dendritic action potentials during an STDP protocol in CA1 pyramidal cells, inactivation of dendritic A-type $\mathrm{K}^{+}$channels is necessary (Watanabe et al., 2002). An increase in $\mathrm{K}^{+}$channel expression with developmental age could also contribute to reducing the back-propagation of single action potentials and thus mean that, in the adult hippocampus, a burst of postsynaptic spikes is necessary for sufficient depolarization during spiketiming-dependent induction of LTP.

What could be the function of such a developmental shift in induction rules for plasticity? In the developing brain, correlated activity appears to be important in refining the synaptic connections in a neuronal network. The occurrence of both LTP and LTD mechanisms at a synapse, dependent on the predictive ("causal") or nonpredictive timing of incoming presynaptic signal, acts to modify synaptic weights. However, in the adult brain, a more refined mechanism of induction of plasticity might be necessary to, for example, prevent random presynaptic and postsynaptic coincident activity from altering patterns of synaptic weights. One proposal is that burst activity plays a role that is different from that of single spiking during memory processing in hippocampal circuitry (Paulsen and Moser, 1998; Pike et al., 1999). In the adult hippocampus, complex spike burst activity is observed during certain behavioral states, including spatial exploration of an environment, when an animal is presumably learning about that environment (O'Keefe, 1976; Buzsaki et al., 2002). Of interest, in vivo electrophysiological studies have reported a reduction in interneuron activity during experience of a novel location in spatial behavioral tasks, with the suggestion that such decreases in inhibition might relate to encoding of new information (Wilson and McNaughton, 1993; Fyhn et al., 2002). One could speculate that the switch in induction rules for LTP that we observe in the adult hippocampus during reduced $\mathrm{GABA}_{\mathrm{A}}$ receptor-mediated inhibition could reflect different encoding rules dependent on the state of the local hippocampal GABAergic network in which those synapses are being modified.

In conclusion, in addition to the extensive changes in synaptic plasticity that occur in very early postnatal development (Yasuda et al., 2003), the present findings demonstrate an additional gradual developmental shift in the induction rules for spike-timingdependent plasticity in the hippocampus from juvenile to adult animals that can be accounted for by increased $\mathrm{GABA}_{\mathrm{A}}$ receptormediated inhibition. Thus, in the mature hippocampal network, it is not only the temporal order of synaptic activation but also the nature of the postsynaptic signal within the local network state that become of crucial importance for synaptic plasticity.

\section{References}

Banks MI, Hardie JB, Pearce RA (2002) Development of GABA(A) receptor-mediated inhibitory postsynaptic currents in hippocampus. J Neurophysiol 88:3097-3107.

Bi GQ, Poo MM (1998) Synaptic modifications in cultured hippocampal neurons: dependence on spike timing, synaptic strength, and postsynaptic cell type. J Neurosci 18:10464-10472.

Bi GQ, Poo MM (2001) Synaptic modification by correlated activity: Hebb's postulate revisited. Annu Rev Neurosci 24:139-166.

Bliss TV, Collingridge GL (1993) A synaptic model of memory: long-term potentiation in the hippocampus. Nature 361:31-39.
Buzsaki G, Csicsvari J, Dragoi G, Harris K, Henze D, Hirase H (2000) Homeostatic maintenance of neuronal excitability by burst discharges in vivo. Cereb Cortex 12:893-899.

Cherubini E, Gaiarsa JL, Ben-Ari Y (1991) GABA: an excitatory transmitter in early postnatal life. Trends Neurosci 14:515-519.

Cohen AS, Lin DD, Coulter DA (2000) Protracted postnatal development of inhibitory synaptic transmission in rat hippocampal area CA1 neurons. J Neurophysiol 84:2465-2476.

Davies CH, Starkey SJ, Pozza MF, Collingridge GL (1991) GABA autoreceptors regulate the induction of LTP. Nature 349:609-611.

Debanne D, Gahwiler BH, Thompson SM (1998) Long-term synaptic plasticity between pairs of individual CA3 pyramidal cells in rat hippocampal slice cultures. J Physiol (Lond) 507:237-247.

Fyhn M, Molden S, Hollup S, Moser MB, Moser E (2002) Hippocampal neurons responding to first-time dislocation of a target object. Neuron 35:555-566.

Ganguly K, Schinder AF, Wong ST, Poo M-M (2001) GABA itself promotes the developmental switch of neuronal GABAergic responses from excitation to inhibition. Cell 105:521-532.

Hebb DO (1949) The organization of behavior. New York: Wiley.

Magee JC, Johnston D (1997) A synaptically controlled, associative signal for Hebbian plasticity in hippocampal neurons. Science 275:209-213.

Markram H, Lubke J, Frotscher M, Sakmann B (1997) Regulation of synaptic efficacy by coincidence of postsynaptic APs and EPSPs. Science 275:213-215.

Martin SJ, Grimwood PD, Morris RG (2000) Synaptic plasticity and memory: an evaluation of the hypothesis. Annu Rev Neurosci 23:649-711.

Morishita W, Alger BE (2001) Direct depolarization and antidromic action potentials transiently suppress dendritic IPSPs in hippocampal CA1 pyramidal cells. J Neurophysiol 85:480-484.

Nishiyama M, Hong K, Mikoshiba K, Poo MM, Kato K (2000) Calcium stores regulate the polarity and input specificity of synaptic modification. Nature 408:584-588.

O'Keefe J (1976) Place units in the hippocampus of the freely moving rat. Exp Neurol 51:78-109.

Paulsen O, Moser EI (1998) A model of hippocampal memory encoding and retrieval: GABAergic control of synaptic plasticity. Trends Neurosci 21:273-278.

Paulsen O, Sejnowski TJ (2000) Natural patterns of activity and long-term synaptic plasticity. Curr Opin Neurobiol 10:172-179.

Pike FG, Meredith RM, Olding AW, Paulsen O (1999) Postsynaptic bursting is essential for "Hebbian" induction of associative long-term potentiation at excitatory synapses in rat hippocampus. J Physiol (Lond) 518:571-576.

Ramon y Cajal S (1894) La fine structure des centres nerveux. Proc R Soc Lond B Biol Sci 55:444-468.

Song S, Miller KD, Abbott LF (2000) Competitive Hebbian learning through spike-timing-dependent synaptic plasticity. Nat Neurosci 3:919-926.

Thomas MJ, Watabe AM, Moody TD, Makhinson M, O’Dell TJ (1998) Postsynaptic complex spike bursting enables the induction of LTP by theta frequency synaptic stimulation. J Neurosci 18:7118-7126.

Tsubokawa H, Ross WN (1996) IPSPs modulate spike backpropagation and associated $\left[\mathrm{Ca}^{2+}\right]_{\mathrm{i}}$ changes in the dendrites of hippocampal CA1 pyramidal neurons. J Neurophysiol 76:2896-2906.

Watanabe S, Hoffman DA, Migliore M, Johnston D (2002) Dendritic K+ channels contribute to spike-timing dependent long-term potentiation in hippocampal pyramidal neurons. Proc Natl Acad Sci USA 99:8366-8371.

Wigström H, Gustafsson B (1983) Facilitated induction of hippocampal longlasting potentiation during blockade of inhibition. Nature 301:603-604.

Wigström H, Gustafsson B, Huang YY, Abraham WC (1986) Hippocampal long-term potentiation is induced by pairing single afferent volleys with intracellularly injected depolarizing current pulses. Acta Physiol Scand 126:317-319.

Wilson MA, McNaughton BL (1993) Dynamics of the hippocampal ensemble code for space. Science 261:1055-1058.

Yasuda H, Barth AL, Stellwagen D, Malenka RC (2003) A developmental switch in the signaling cascades for LTP induction. Nat Neurosci 6:15-16.

Zhang L, Spigelman I, Carlen PL (1991) Development of GABA-mediated, chloride-dependent inhibition in CA1 pyramidal neurones of immature rat hippocampal slices. J Physiol (Lond) 444:25-49.

Zhang LI, Tao HW, Holt CE, Harris WA, Poo M (1998) A critical window for cooperation and competition among developing retinotectal synapses. Nature 395:37-44. 